\title{
RELAÇÃO ENTRE MÁ OCLUSÃo E HÁBITOS ORAIS EM RESPIRADORES ORAIS
}

\section{Relationship between malocclusion and oral habits in mouth breathing}

\author{
Flávia Leães de Almeida ${ }^{(1)}$, Ana Maria Toniolo da Silva ${ }^{(2)}$, Eliane de Oliveira Serpa ${ }^{(3)}$
}

\begin{abstract}
RESUMO
Objetivo: verificar a relação entre má oclusão e hábitos orais deletérios em um grupo de respiradores orais. Métodos: estudo retrospectivo realizado por meio da análise de dados dos prontuários de 41 crianças, com idades entre sete e 12 anos, sendo 21 do sexo masculino e 20 do sexo feminino, todas respiradoras orais. As informações coletadas foram organizadas em um Banco de Dados no programa Excel, considerando-se as variáveis: idade, sexo, presença e tipo de hábito oral, e presença e tipo de má oclusão. Resultados: todas as crianças apresentavam algum tipo de má oclusão, com predomínio de classe II de Angle, e sobressaliência acentuada; entre os hábitos, o mais incidente foi o de colocação de objetos na boca, embora todas as crianças tenham apresentado um, ou mais hábitos deletérios; no cruzamento das variáveis, a única relação estatisticamente significante encontrada foi entre o hábito de lamber lábios e a sobressaliência acentuada. Conclusão: conclui-se que, nessa amostra, a presença de hábitos deletérios não foi determinante para a instalação das más oclusões, que a respiração oral pode ter desencadeado as más oclusões nesse grupo e que, a associação dos hábitos deletérios com a respiração oral, pode ter agido como fator agravante para a instalação, ou desenvolvimento das más oclusões nessas crianças.
\end{abstract}

DESCRITORES: Respiração Bucal; Má Oclusão; Hábitos

\section{INTRODUÇÃO}

A função da respiração é considerada vital para o bom funcionamento do organismo e, desde o nascimento, exerce influência na manutenção da organização esquelética, dentária e muscular do Sistema Estomatognático, contribuindo, dessa forma, para o adequado desenvolvimento das funções orofaciais e para o crescimento esquelético harmonioso. A presença de um impedimento à respiração nasal,

(1) Fonoaudióloga; Especialista em Fonoaudiologia pela Universidade Federal de Santa Maria, UFSM, Santa Maria, RS; Mestranda no Programa de Pós-Graduação em Distúrbios da Comunicação Humana.

(2) Fonoaudióloga; Professora Associada do Departamento de Fonoaudiologia da Universidade Federal de Santa Maria, UFSM, Santa Maria, RS; Docente do Programa de PósGraduação em Distúrbios da Comunicação Humana da Universidade Federal de Santa Maria, UFSM, Santa Maria, RS; Doutora em Ciências dos Distúrbios da Comunicação Humana pela Universidade Federal de São Paulo.

(3) Ortodontista; Clinica Odontológica na cidade de Santa Maria, UFSM, Santa Maria, RS; Mestre em Distúrbios da Comunicação Humana pela Universidade Federal de Santa Maria, UFSM, Santa Maria, RS. poderá gerar um padrão de suplência oral e, caso esse se instale durante o período de desenvolvimento, poderá determinar diversas alterações que prejudicam, significativamente, esse Sistema ${ }^{1-5}$.

Distúrbios respiratórios, como a respiração oral, estão diretamente relacionados às alterações craniofaciais e oclusais, podendo ser decorrentes da presença de hábitos orais deletérios, ou mesmo serem considerados como um deles ${ }^{6,7}$.

Os hábitos deletérios são, comumente, definidos como padrões neuromusculares atípicos que podem determinar, entre outras coisas, o desenvolvimento das más oclusões, devido aos prejuízos que ocasionam, principalmente, na posição dos dentes, desencadeando alterações, também, no Sistema Estomatognático, pois introduzem forças estranhas a esse. Esses podem ter origem fisiológica; emocional ou aprendida e seus prejuízos serão determinados, em especial, pela frequência, intensidade, duração e o objeto utilizado, bem como, a idade do sujeito na época de instalação dos hábitos ${ }^{8-11}$.

Dentre os hábitos deletérios mais comentados na literatura, a sucção prolongada de dedo, chupeta ou mamadeira, frequentemente, encontra-se associada 
à etiologia das más oclusões, fato esse que, de forma alguma, exclui a relação dos outros hábitos existentes com os diversos tipos de más oclusões ${ }^{12-15}$. Porém, a ocorrência de hábitos orais, independente do tipo, é um fator prejudicial para o adequado desenvolvimento da oclusão e das funções do Sistema Estomatognático, contribuindo, inclusive, para o desenvolvimento da respiração oral ${ }^{16}$.

Com base nestas colocações esse estudo procurou verificar a possível relação entre má oclusão e hábitos orais deletérios em um grupo de respiradores orais.

\section{MÉTODOS}

Este estudo caracteriza-se por ser de caráter observacional, exploratório, quantitativo, transversal, e retrospectivo, visto que os dados foram retirados de prontuários.

Foram coletados os dados de 41 crianças, que participaram do projeto "Estudo da relação entre Respiração Oronasal, tipo facial e espaço nasofaríngeo: uma abordagem multidisciplinar", vinculado ao Setor de Triagem do Serviço de Atendimento Fonoaudiológico (SAF) da Universidade Federal de Santa Maria (UFSM), o qual foi concluído no ano de 2002.

Os critérios de inclusão para a participação na pesquisa foram os que seguintes: as crianças deveriam estar em fase de dentição mista, não poderiam ter realizado qualquer tratamento ortodôntico e deveriam ser respiradoras orais.

A amostra foi composta por 41 crianças com idades variando entre 07 e 12 anos, sendo $21(51,21 \%)$ do sexo masculino e 20 (48,79\%) do sexo feminino que tinham como queixa principal dificuldade respiratória, sendo essa relatada pelos pais, ou responsáveis. Participaram da pesquisa apenas as crianças em que os pais e/ou responsáveis foram favoráveis ao termo de Consentimento Livre e Esclarecido (TCLE) elaborado pela pesquisadora.

Os dados utilizados na pesquisa foram retirados da anamnese inicial e das avaliações Fonoaudiológica, Otorrinolaringológica e Ortodôntica que procuraram investigar, entre outros aspectos, presença/ ausência, e tipo de hábitos orais (tanto intra, como extra-orais); definição do padrão respiratório, tipo de patologia nasorrespiratória; e tipo de oclusão dentária.

A avaliação fonoaudiológica foi realizada por uma professora do curso de Fonoaudiologia da UFSM, e constou de anamnese e exame orofacial. Estas avaliações foram baseadas no Protocolo de Avaliação Fonoaudiológica do Sistema Estomatognático utilizado no SAF - UFSM. A anamnese teve como principais objetivos identificar a queixa principal e obter informações sobre antecedentes pessoais, estado de saúde, hábitos alimentares anteriores e atuais, sono, desenvolvimento da fala, existência e tipo de hábitos orais deletérios e alterações respiratórias. No exame orofacial, foram avaliados aspectos com relação à postura de repouso e tonicidade dos órgãos fonoarticulatórios, e o comportamento destas estruturas durante as funções de sucção, mastigação, deglutição, respiração, contribuindo, com os dados obtidos, para o diagnóstico de respiração oral.

A avaliação otorrinolaringológica foi realizada por um médico Otorrinolaringologista, colaborador do projeto, e constou de anamnese e exame clínico (baseados no protocolo de avaliação otorrinolaringológica do SAF-UFSM), além de otoscopia, rinoscopia, e rinofaringoscopia. Todas as avaliações otorrinolaringológicas tiveram como objetivo confirmar a queixa de respiração oral e investigar as prováveis patologias nasorrespiratórias associadas.

A avaliação ortodôntica, realizada pela ortodontista responsável pelo projeto inicial de onde os dados foram coletados, constou de exame clínico, onde se observou o tipo de dentição (decídua, mista ou permanente) e de oclusão (classe I, II ou III) conforme classificação de Angle ${ }^{17}$, além de sobressaliência acentuada e sobremordida. Considerou-se sobressaliência o aumento do trespasse dos incisivos superiores a frente dos inferiores no plano horizontal, e sobremordida um trespasse maior que $1 / 3$ dos incisivos superiores sobre os inferiores no plano vertical.

Os dados que serão expostos, no capítulo dos resultados, nas Tabelas 1 e 2 apresentam o número total de crianças de acordo com as variáveis pesquisadas; a Tabela 3 apresenta a relação entre as más oclusões classes I, II e III de Angle, com a presença/ ausência dos hábitos estudados, na qual não foi possível aplicar nenhum teste estatístico devido às baixas frequências apresentadas; os dados mostrados na Tabela 4 expõem a relação entre a presença/ ausência de sobressaliência acentuada (trespasse horizontal) e de sobremordida (trespasse vertical), com a presença/ ausência dos hábitos estudados.

O projeto que deu origem a esse trabalho foi registrado no Gabinete de Projetos da Universidade Federal de Santa Maria - UFSM, sob número 5859.

As informações colhidas foram organizadas em um Banco de Dados no programa Excel, considerando-se as variáveis: idade, sexo, presença e tipo de hábito oral, e presença e tipo de má oclusão. Para a avaliação estatística utilizou-se o Teste Nãoparamétrico Qui-Quadrado, considerando-se o nível de significância $p<0,05$. 
Tabela 1 - Distribuição absoluta e relativa das 41 crianças respiradoras orais segundo classificação da má oclusão, conforme Angle, Sobressaliência acentuada e Sobremordida

\begin{tabular}{lcc}
\hline \multirow{2}{*}{ Má Oclusão } & \multicolumn{2}{c}{ Crianças respiradoras orais } \\
\cline { 2 - 3 } & Absoluto $(\mathbf{n})$ & Relativo $(\%)$ \\
\hline Classe I & 16 & 39,02 \\
Classe II & 24 & 58,54 \\
Classe III & 1 & 2,44 \\
Sobressaliência acentuada & 26 & 63,41 \\
Sobremordida & 12 & 29,27 \\
\hline
\end{tabular}

Tabela 2 - Distribuição absoluta e relativa das 41 crianças respiradoras orais segundo os hábitos orais apresentados

\begin{tabular}{lcc}
\hline \multirow{2}{*}{ Hábitos } & \multicolumn{2}{c}{ Crianças respiradoras orais } \\
\cline { 2 - 3 } & Absoluto $(\mathbf{n})$ & Relativo (\%) \\
\hline Apoio de Cabeça & 12 & 29,27 \\
Bruxismo & 11 & 26,83 \\
Lamber Lábios & 13 & 31,71 \\
Movimentos anormais de Língua & 4 & 9,76 \\
Morder Lábios e Bochechas & 9 & 21,95 \\
Sucção de Dedo & 7 & 17,07 \\
Sucção de Chupeta & 8 & 19,51 \\
Sucção de Mamadeira & 9 & 21,95 \\
Colocação de Objetos na Boca & 20 & 48,78 \\
Onicofagia & 15 & 36,59 \\
\hline
\end{tabular}

\section{RESULTADOS}

Com relação à variável oclusão, verificou-se que, de acordo com a classificação de Angle, a classe II foi o tipo de má oclusão mais frequente, sendo encontrada em $24(58,54 \%)$ das crianças. A sobressaliência acentuada foi encontrada em $26(63,41 \%)$ crianças, e a sobremordida em $12(29,27 \%)$ crianças (Tabela 1).

Quanto aos hábitos orais, houve predomínio do hábito de colocar objetos na boca, ocorrendo em 20 crianças $(48,78 \%)$. O hábito menos frequente foi o de movimentos anormais de língua, sendo observado em 4 crianças $(9,76 \%)$.

Os dados da Tabela 3 demonstraram que 0 predomínio dos resultados, tanto para a presença, quanto para a ausência da maioria dos hábitos orais ocorreu, em crianças portadoras de má oclusão classe II e classe I. No cruzamento do hábito de sucção de mamadeira, se observou um discreto predomínio da má oclusão classe I.

Os resultados expostos na Tabela 4 demonstram que, com relação à presença/ausência de sobressaliência acentuada e hábitos orais, houve semelhança na maioria dos achados, uma vez que não apresentaram significância estatística ( $p>$ $0,05)$ e, ainda, porque a maior ocorrência de crianças que tinham, e que não tinham os hábitos em questão, eram portadoras de trespasse horizontal. Excetuam-se as relações entre sobressaliência acentuada e bruxismo, e da mesma com a sucção de mamadeira, que, apesar de não ter apresentado significância estatística ( $p>0,05)$, demonstrou a prevalência da sobressaliência acentuada entre as crianças que não praticavam os hábitos, acontecendo o contrário, ainda que de forma discreta, entre as crianças que os apresentavam; e, também, a relação entre o trespasse horizontal com o hábito de lamber lábios, que apresentou resultado estatisticamente significante $(p<0,05)$.

No cruzamento das variáveis sobremordida e hábitos, independentemente do tipo de hábito oral abordado, o predomínio dos resultados, tanto na presença quanto na ausência dos mesmos, não apresentaram significância estatística $(p>0,05)$ (Tabela 4). 
Tabela 3 - Distribuição absoluta e relativa, respectivamente, das 41 crianças respiradoras orais relacionando a presença e a ausência dos hábitos com os tipos de má oclusão, conforme classificação de Angle

\begin{tabular}{|c|c|c|c|c|c|c|}
\hline \multirow{3}{*}{ Hábito } & \multicolumn{6}{|c|}{ Má Oclusão } \\
\hline & \multicolumn{2}{|c|}{ Classe I } & \multicolumn{2}{|c|}{ Classe II } & \multicolumn{2}{|c|}{ Classe III } \\
\hline & $\mathbf{N}$ & $S$ & $\mathbf{N}$ & $\mathrm{S}$ & $\mathbf{N}$ & $\mathbf{S}$ \\
\hline \multirow{2}{*}{ Apoio de Cabeça } & $\mathrm{n}=14$ & $\mathrm{n}=2$ & $\mathrm{n}=14$ & $\mathrm{n}=10$ & $\mathrm{n}=1$ & $\mathrm{n}=0$ \\
\hline & $34,15 \%$ & $4,48 \%$ & $34,15 \%$ & $24,39 \%$ & $2,44 \%$ & $0,00 \%$ \\
\hline \multirow{2}{*}{ Bruxismo } & $\mathrm{n}=12$ & $\mathrm{n}=4$ & $\mathrm{n}=17$ & $n=7$ & $\mathrm{n}=1$ & $\mathrm{n}=0$ \\
\hline & $29,27 \%$ & $9,73 \%$ & $41,46 \%$ & $17,7 \%$ & $2,44 \%$ & $0,00 \%$ \\
\hline \multirow{2}{*}{ Lamber Lábios } & $\mathrm{n}=11$ & $n=5$ & $\mathrm{n}=17$ & $n=7$ & $\mathrm{n}=0$ & $n=1$ \\
\hline & $26,83 \%$ & $12,20 \%$ & $41,46 \%$ & $17,07 \%$ & $0,00 \%$ & $2,44 \%$ \\
\hline \multirow{2}{*}{ Movimentos anormais língua } & $\mathrm{n}=16$ & $\mathrm{n}=0$ & $\mathrm{n}=20$ & $\mathrm{n}=4$ & $\mathrm{n}=1$ & $\mathrm{n}=0$ \\
\hline & $39,02 \%$ & $0,00 \%$ & $48,78 \%$ & $9,73 \%$ & $2,44 \%$ & $0,00 \%$ \\
\hline \multirow{2}{*}{ Morder Lábios e Bochechas } & $\mathrm{n}=14$ & $\mathrm{n}=2$ & $\mathrm{n}=17$ & $\mathrm{n}=7$ & $\mathrm{n}=1$ & $\mathrm{n}=0$ \\
\hline & $34,15 \%$ & $4,88 \%$ & $41,46 \%$ & $17,07 \%$ & $2,44 \%$ & $0,00 \%$ \\
\hline \multirow{2}{*}{ Sucção de Dedo } & $\mathrm{n}=14$ & $\mathrm{n}=2$ & $\mathrm{n}=19$ & $n=5$ & $\mathrm{n}=1$ & $\mathrm{n}=0$ \\
\hline & $34,15 \%$ & $4,88 \%$ & $46,34 \%$ & $12,20 \%$ & $2,44 \%$ & $0,00 \%$ \\
\hline \multirow{2}{*}{ Sucção de Chupeta } & $\mathrm{n}=12$ & $\mathrm{n}=4$ & $\mathrm{n}=20$ & $n=4$ & $\mathrm{n}=1$ & $\mathrm{n}=0$ \\
\hline & $29,27 \%$ & $9,73 \%$ & $48,78 \%$ & $9,73 \%$ & $2,44 \%$ & $0,00 \%$ \\
\hline \multirow{2}{*}{ Sucção de Mamadeira } & $\mathrm{n}=11$ & $n=5$ & $\mathrm{n}=20$ & $\mathrm{n}=4$ & $\mathrm{n}=1$ & $\mathrm{n}=0$ \\
\hline & $26,83 \%$ & $12,20 \%$ & $48,78 \%$ & $9,73 \%$ & $2,44 \%$ & $0,00 \%$ \\
\hline \multirow{2}{*}{ Colocação de Objetos na Boca } & $n=8$ & $n=8$ & $\mathrm{n}=12$ & $\mathrm{n}=12$ & $\mathrm{n}=1$ & $\mathrm{n}=0$ \\
\hline & $19,51 \%$ & $19,51 \%$ & $29,27 \%$ & $29,27 \%$ & $2,44 \%$ & $0,00 \%$ \\
\hline \multirow{2}{*}{ Onicofagia } & $\mathrm{n}=11$ & $\mathrm{n}=5$ & $\mathrm{n}=14$ & $\mathrm{n}=10$ & $\mathrm{n}=1$ & $\mathrm{n}=0$ \\
\hline & $26,83 \%$ & $12,20 \%$ & $34,15 \%$ & $24,39 \%$ & $2,44 \%$ & $0,00 \%$ \\
\hline
\end{tabular}

\section{DISCUSSÃO}

A análise dos resultados da Tabela 1 mostra a estreita relação que se estabelece entre respiração oral e má oclusão neste grupo. Ainda que os resultados demonstrem o predomínio da má oclusão do tipo classe II e da sobressaliência acentuada, é possível observar que todas as 41 crianças apresentavam algum tipo de má oclusão, inclusive demonstrando indícios da existência concomitante de mais de um tipo de má oclusão em algumas crianças. Corroboram estes achados os resultados de pesquisas que relacionaram o equilíbrio dentário e craniofacial com a respiração e referiram que o primeiro fator se torna dependente do segundo, ou seja, um mau desenvolvimento da oclusão ou da forma maxilo-mandibular pode estar diretamente associado à presença de uma patologia respiratória como a respiração oral, além de verificarem a associação da má oclusão classe II com a respiração oral e o predomínio de sobressaliência em crianças em fase de dentição mista ${ }^{18-20}$. Porém, vão de encontro a estes achados, resultados de predomínio da má oclusão classe I sobre as demais más oclusões em escolares ${ }^{21,22}$.

Considerando-se os dados evidenciados na Tabela 2, observa-se que dos 10 hábitos encontrados no grupo de crianças, o que apresentou maior incidência foi o de colocação de objetos na boca, seguido de onicofagia, lamber lábios, apoio de cabeça, bruxismo, sucção de mamadeira e morder lábios e bochechas, sucção de chupeta, sucção de dedo e, por fim, o menos incidente foi o hábito de movimentos anormais de língua. Além disso, a exposição destes resultados demonstra que as crianças do estudo devem ter realizado mais de um hábito simultaneamente, pois o número de hábitos encontrados (108) foi superior ao número de sujeitos da amostra (41) o que é um fator agravante, visto que todos os participantes do estudo são portadores de algum tipo de má oclusão e de respiração oral. Este resultado contrasta com o que, comumente, é evidenciado na literatura, pois estudos demonstraram que os hábitos mais frequentemente encontrados em indivíduos com o perfil semelhante ao da amostra desse estudo são os hábitos de sucção prolongados ${ }^{13,22-24}$. 
Tabela 4 - Distribuição absoluta e relativa, respectivamente, das 41 crianças respiradoras orais relacionando a presença e a ausência dos hábitos com a presença e a ausência de sobressaliência acentuada e sobremordida

\begin{tabular}{|c|c|c|c|c|c|}
\hline \multirow{3}{*}{ Hábito } & & \multicolumn{4}{|c|}{ Má Oclusão } \\
\hline & & \multicolumn{2}{|c|}{ Sobressaliência Acentuada } & \multicolumn{2}{|c|}{ Sobremordida } \\
\hline & & $\mathbf{N}$ & $\mathbf{S}$ & $\mathbf{N}$ & $\mathbf{s}$ \\
\hline \multirow{5}{*}{ Apoio de Cabeça } & \multirow{2}{*}{ N } & $\mathrm{n}=11$ & $\mathrm{n}=18$ & $\mathrm{n}=20$ & $\mathrm{n}=9$ \\
\hline & & $26,83 \%$ & $43,90 \%$ & $48,78 \%$ & $21,95 \%$ \\
\hline & \multirow{3}{*}{$\mathbf{s}$} & $\mathrm{n}=4$ & $\mathrm{n}=8$ & $n=9$ & $n=3$ \\
\hline & & $9,73 \%$ & $19,51 \%$ & $21,95 \%$ & $7,32 \%$ \\
\hline & & & & \multicolumn{2}{|c|}{${ }^{*} p=0.1493$} \\
\hline \multirow{5}{*}{ Bruxismo } & \multirow{2}{*}{$\mathbf{N}$} & $n=9$ & $\mathrm{n}=21$ & $\mathrm{n}=23$ & $\mathrm{n}=7$ \\
\hline & & $21,95 \%$ & $51,22 \%$ & $56,10 \%$ & $17,7 \%$ \\
\hline & \multirow{3}{*}{$\mathbf{S}$} & $\mathrm{n}=6$ & $\mathrm{n}=5$ & $\mathrm{n}=6$ & $\mathrm{n}=5$ \\
\hline & & $14,63 \%$ & $12,20 \%$ & $14,63 \%$ & $12,20 \%$ \\
\hline & & \multicolumn{2}{|c|}{${ }^{*} p=2.0901$} & \multicolumn{2}{|c|}{${ }^{*} p=1.9026$} \\
\hline \multirow{5}{*}{ Lamber Lábios } & \multirow{2}{*}{$\mathbf{N}$} & $\mathrm{n}=10$ & $\mathrm{n}=18$ & $\mathrm{n}=21$ & $\mathrm{n}=7$ \\
\hline & & $24,39 \%$ & $43,90 \%$ & $51,22 \%$ & $17,7 \%$ \\
\hline & \multirow{3}{*}{$\mathbf{S}$} & $\mathrm{n}=5$ & $\mathrm{n}=8$ & $\mathrm{n}=8$ & $n=5$ \\
\hline & & $12,20 \%$ & $19,51 \%$ & $19,51 \%$ & $12,20 \%$ \\
\hline & & \multicolumn{2}{|c|}{${ }^{*} p=0.0289$} & \multicolumn{2}{|c|}{${ }^{*} p=0.7771$} \\
\hline \multirow{5}{*}{$\begin{array}{l}\text { Movimentos anormais } \\
\text { língua }\end{array}$} & \multirow{3}{*}{$\mathbf{N}$} & $n=14$ & $n=23$ & $n=25$ & $n=12$ \\
\hline & & $\%=34,15$ & $\%=56,10$ & $\%=60,98$ & $\%=29,27$ \\
\hline & & $\mathrm{n}=1$ & $n=3$ & $\mathrm{n}=4$ & $\mathrm{n}=0$ \\
\hline & 8 & $2,44 \%$ & $7,32 \%$ & $9,76 \%$ & $0,00 \%$ \\
\hline & & & & & \\
\hline & & $\mathrm{n}=12$ & $\mathrm{n}=20$ & $n=23$ & $n=9$ \\
\hline & $\mathbf{N}$ & $29,27 \%$ & $48,78 \%$ & $56,10 \%$ & $21,95 \%$ \\
\hline Morder Lábios e & & $\mathrm{n}=3$ & $\mathrm{n}=6$ & $\mathrm{n}=6$ & $\mathrm{n}=3$ \\
\hline & 5 & $7,32 \%$ & $14,63 \%$ & $14,63 \%$ & $7,32 \%$ \\
\hline & & & & & \\
\hline & & $\mathrm{n}=14$ & $\mathrm{n}=20$ & $\mathrm{n}=25$ & $\mathrm{n}=9$ \\
\hline & $\mathbf{N}$ & $34,15 \%$ & $48,78 \%$ & $60,98 \%$ & $21,95 \%$ \\
\hline Sucção de Dedo & $S$ & $\mathrm{n}=1$ & $\mathrm{n}=6$ & $\mathrm{n}=4$ & $n=3$ \\
\hline & 3 & $2,44 \%$ & $14,63 \%$ & $9,73 \%$ & $7,32 \%$ \\
\hline & & & & & \\
\hline & $\mathrm{N}$ & $\mathrm{n}=11$ & $n=22$ & $\mathrm{n}=23$ & $n=10$ \\
\hline & $\mathbf{N}$ & $26,83 \%$ & $53,66 \%$ & $56,10 \%$ & $24,39 \%$ \\
\hline Sucção de Chupeta & $s$ & $\mathrm{n}=4$ & $\mathrm{n}=4$ & $\mathrm{n}=6$ & $\mathrm{n}=2$ \\
\hline & 0 & $9,73 \%$ & $9,73 \%$ & $14,63 \%$ & $4,88 \%$ \\
\hline & & & & & \\
\hline & & $\mathrm{n}=10$ & $\mathrm{n}=22$ & $\mathrm{n}=23$ & $n=9$ \\
\hline & $\mathbf{N}$ & $24,39 \%$ & $53,66 \%$ & $56,10 \%$ & $21,95 \%$ \\
\hline Sucção de Mamadeira & & $\mathrm{n}=5$ & $\mathrm{n}=4$ & $\mathrm{n}=6$ & $\mathrm{n}=3$ \\
\hline & S & $12,20 \%$ & $9,73 \%$ & $14,63 \%$ & $7,32 \%$ \\
\hline & & & & & \\
\hline & & $n=9$ & $n=12$ & $\mathrm{n}=16$ & $\mathrm{n}=5$ \\
\hline & $\mathbf{N}$ & $21,95 \%$ & $29,27 \%$ & $39,02 \%$ & $12,20 \%$ \\
\hline Colocação de Objetos & & $\mathrm{n}=6$ & $n=14$ & $n=13$ & $\mathrm{n}=7$ \\
\hline & 5 & $14,63 \%$ & $34,15 \%$ & $31,71 \%$ & $17,7 \%$ \\
\hline & & & & & \\
\hline & $N$ & $\begin{array}{c}\mathrm{n}=9 \\
21,95 \%\end{array}$ & $\begin{array}{c}\mathrm{n}=17 \\
41,46 \%\end{array}$ & $\begin{array}{c}n=19 \\
46,34 \%\end{array}$ & $\begin{array}{c}\mathrm{n}=7 \\
17,7 \%\end{array}$ \\
\hline Onicofagia & $c$ & $\mathrm{n}=6$ & $n=9$ & $n=10$ & $\mathrm{n}=5$ \\
\hline & 5 & $14,63 \%$ & $21,95 \%$ & $24,39 \%$ & $12,20 \%$ \\
\hline & & & & & \\
\hline
\end{tabular}


Os dados apresentados na Tabela 3 mostram que a presença ou ausência de uma variável, não exclui a ocorrência, ou não-ocorrência da outra variável, ou seja, os hábitos orais não influenciaram no desenvolvimento das más oclusões classes I, II e III nesse grupo. Outro fator relevante na análise destes resultados, é que como todos os participantes do estudo são respiradores orais acredita-se que, tanto os hábitos orais, quanto às más oclusões sofreram influência desta patologia nesta amostra, concordando com outros achados que evidenciaram associação entre respiração oral, alterações dentárias e hábitos orais deletérios ${ }^{23,25}$.

Com relação ao resultado encontrado no cruzamento do hábito de sucção de mamadeira com as classes I, II e III (Tabela 3), pode-se dizer que o uso prolongado da mamadeira, na maioria das vezes, afeta de maneira significativa a oclusão, podendo, inclusive ser o fator desencadeante de alterações na mordida e na chave de molar dos indivíduos que o praticam, porém este resultado surpreende positivamente, visto que o predomínio do hábito ocorreu em crianças classe I, sugerindo que os prejuízos não foram tão expressivos. Esses achados vão de encontro aos de estudos que relacionaram tipo de aleitamento e hábitos deletérios com a ocorrência de más oclusões e encontraram associação significativa entre a prática de hábitos com a presença de má oclusão, com a particularidade da maior incidência das más oclusões serem alterações na mordida dos tipos cruzada e/ou aberta ${ }^{14,15,26}$.

Os dados expostos na Tabela 4 reafirmam que neste grupo de 41 crianças, a prática, ou não de hábitos orais não foi um fator determinante no desenvolvimento das más oclusões, neste caso, da sobressaliência acentuada e da sobremordida, contrapondo-se a outros resultados, onde foram evidenciadas relações significantes entre a ocorrência de más oclusões e a presença de hábitos orais deletérios em diferentes tipos de população 11,27,28. Porém, assim como nesse estudo, foi observado um alto índice de hábitos orais em adolescentes, sem que ficasse constatado que a prática dos mesmos tivesse relação direta com a presença de má oclusão, inclusive sendo encontrado um número superior de hábitos orais em sujeitos com oclusão normal ${ }^{29}$.

Ainda na Tabela 4, verifica-se relação estatisticamente significante $(p<0,05)$ entre o hábito de lamber lábios com a sobressaliência acentuada. $\mathrm{A}$ realização desse hábito é uma característica, frequentemente, encontrada nos respiradores orais, pois o ressecamento dos lábios, ocasionado pela permanência da boca aberta, leva os portadores de respiração oral a umidificá-los frequentemente, o que acaba por estimular o hábito, gerando, dessa forma, um ciclo de ações nocivas às estruturas envolvidas. Ao contrário desse estudo, em pesquisa realizada com crianças de 3 a 5 anos de idade, foi verificada relação positiva entre a ocorrência de má oclusão e de hábitos deletérios, estando a sobremordida presente em $19,8 \%$ da amostra, e a sucção de chupeta, como hábito deletério mais incidente, em $65,4 \%$ das crianças ${ }^{28}$.

Dessa forma, foi possível verificar que, os hábitos orais deletérios não foram os fatores desencadeantes do desenvolvimento das más oclusões apresentadas pela amostra estudada, porém a presença da respiração oral, também considerada como um hábito deletério, pode ter sido, associada aos demais hábitos, o determinante ou agravante da presença das más oclusões dos participantes desse estudo.

\section{CONCLUSÃO}

Ao término desse estudo, que teve como objetivo verificar a relação entre hábitos orais deletérios e má oclusão em respiradores orais, pôde-se constatar que os hábitos deletérios apresentados não foram fatores determinantes na instalação, ou desenvolvimento das más oclusões. 


\section{ABSTRACT}

Purpose: to check the relationship between malocclusion and deleterious oral habits in a group of mouth breathing. Methods: this study was accomplished through data analysis of 41 children handbooks, with ages between 7 and 12 years, being 21 of masculine gender and 20 of feminine gender, all mouth breathing. The collected information was organized in a database using an Excel program, considering the variables: age, gender, presence and type of oral habit, and presence and type of malocclusion. Results: all children presented some type of malocclusion, with prevalence of class II of Angle, and accentuated overjet; among the habits, the more incident was placement of objects in the mouth, although all children have showed one, or more deleterious habits; in crossing the variables, the only significant statistical relationship found was between the habit of licking lips and accentuated overjet. Conclusions: we may conclude that, in this sample, the presence of deleterious habits were not decisive for installation of malocclusions, that the mouth breathing might have unchained the malocclusions in this group and that, the association of the deleterious habits with the mouth breathing, might have acted as aggravating factor for the installation, or development of the malocclusions in these children.

KEYWORDS: Mouth Breathing; Malocclusion; Habits

\section{REFERÊNCIAS}

1. Linder-Aronson S. Respiratory function in relation to facial morphology and the dentition. Br J Orthod. 1979; 6(2):59-71.

2. Aragão W. Aragão's function regulator, the stomatognathic system and postural changes in children. J Clin Pediatr Dent. 1991; 15(4):226-31.

3. Cardoso M, ProffitWR. Ortodontia contemporânea. Rio de Janeiro: Guanabara Koogan; 1995.

4. Ribeiro EC, Marchiori SC, Silva AMT. Electromyographics muscle EMG activity in mouth and nasal breathing children. J Craniomandib Pract. 2004; 22(2):145-50.

5. Lemos CM, Junqueira PAS, Gomez MVSG, Faria MEJ, Basso SC. Estudo da relação entre a oclusão dentária e a deglutição no respirador oral. Arq Int Otorrinolaringol. 2006; 10(2):114-8.

6. Lino AP. Ortodontia Preventiva Básica. São Paulo: Artes Médicas; 1992.

7. Marchesan IQ. Motricidade oral: visão clínica do trabalho fonoaudiológico integrado com outras especialidades. São Paulo: Pancast; 1993.

8. Enlow DH. Manual sobre crescimento facial. Buenos Aires: Inter-Médica; 1982.

9. Moresca CA, Feres MA. Hábitos viciosos bucais. In: Petrelli E. Ortodontia para fonoaudiologia. São Paulo: Lovise; 1994. 174 p.

10. Onyeaso CO, Sote EO. Prevalence of oral habits in 563 Nigerian preschool children age 3-5 years. Niger Postgrad Med J. 2001; 8(4):193-5.
11. Da Costa OO, Orenuga OO. Dentofacial anomalies related to the digit sucking habit. Afr $\mathrm{J}$ Med Med Sci. 2002; 31(3):239-42.

12. Hanson ML, Cohen MS. Effects of form and function on swallowing and the developing dentition. Am J Orthod. 1973; 64(1):63-82.

13. Amary ICM, Rossi LAF, Yumoto VA, AssencioFerreira VJ, Marchesan IQ. Hábitos deletérios: alterações de oclusão. Rev CEFAC. 2002; 4(2):123-6.

14. Sousa FRN, Taveira GS, Almeida RVD, Padilha WWN. O aleitamento materno e sua relação com hábitos deletérios e maloclusão dentária. Pesq Bras Odontoped Clin Integr. 2004; 4(3):211-6.

15. Souza DFRK, Valle MAS, Pacheco MCT. Relação clínica entre hábitos de sucção, má oclusão, aleitamento e grau de informação prévia das mães. Dent Press Ortodon Ortop Facial. 2006; 11(6):81-90.

16. Bittencourt LP, Modesto A, Bastos EP. Influência do aleitamento sobre a freqüência dos hábitos de sucção. Rev Bras Ortop. 2001; 58(3):191-3.

17. Angle EH. Malooclusion of the teeth. 7. ed. Philadelphia: White Dental Manufacturing Co; 1907.

18. Marchesan IQ, Zorzi J. Anuário CEFAC de Fonoaudiologia. 1999/2000 vol. 1. Rio de Janeiro: Revinter; 2000.

19. Andrade FV, Andrade DV, Araújo AS, Ribeiro ACC, Deccax LDG, Nemr K. Alterações estruturais de órgãos fonoarticulatórios e más oclusões dentárias em respiradores orais de 6 A 10 Anos. Rev CEFAC. 2005; 7(3):318-25. 
20. Emmerich A, Fonseca L, Elias AM, Medeiros UV. Relação entre hábitos bucais, alterações oronasofaringianas e mal-oclusões em préescolares de Vitória, Espírito Santo, Brasil. Cad Saúde Pública. 2004; 20(3):689-97.

21. Zanetti GA. Características da dentadura mista em crianças brasileiras [dissertação]. Bauru (SP): Faculdade de Odontologia de Bauru da Universidade de São Paulo; 2003. Disponível em:URL: http:// www.teses.usp.br/teses/disponiveis/25/25133/tde04042005-163037/.

22. Maciel CTV, Leite ICG. Aspectos etiológicos da mordida aberta anterior e suas implicações nas funções orofaciais. Pró-Fono. 2005; 17(3):293-302.

23. Ribeiro F, Mesquita MCM, Assencio-Ferreira VJ. Respiração oral: alterações oclusais e hábitos orais. Rev CEFAC. 2002; 4(3):187-90.

24. Galvão ACUR, Menezes SFL, Nemr K. Correlação de hábitos orais deletérios entre crianças de 4:00 a 6:00 anos de escola pública e escola particular da cidade de Manaus - AM. Rev CEFAC. 2006; 8(3):328-36.
25. Rejman R, Martins DR, Scavone Júnior H, CotrimFerreira FA, Vellini-Ferreira F. Estudo comparativo das dimensões transversais dos arcos dentários entre jovens com oclusão normal e má oclusão de Classe II, 1 a divisão. Dental Press Ortodon Ortop Facial. 2006; 11(4):118-25.

26. Oliveira AB, Souza FP, Chiappetta ALML. Relação entre hábitos de sucção não-nutritiva, tipo de aleitamento e más oclusões em crianças com dentição decídua. Rev CEFAC. 2006; 8(3):352-9. 27. Santos-Pinto A, Paulin RF, Nakiri JH. Hábitos bucais como fatores etiológicos no desenvolvimento das más oclusões. Rev Assoc Paulist Espec Ortodon Ortop Facial. 2003; 1(1):17-21.

28. Bezerra PKM, Cavalcanti AL, Bezerra PM, Moura C. Maloclusões, tipos de aleitamento e hábitos bucais deletérios em pré-escolares: um estudo de associação. Pesq Bras Odontoped Clin Integr. 2005; 5(3):267-74.

29. Santos AG, Valente SV. Prevalência de hábitos orais deletérios e má oclusão em um Grupo de adolescentes. Rev CEFAC. 2003; 5(3):213-9.

DOI: 10.1590 / S1516- 18462009005000005

RECEBIDO EM: 13/09/2007

ACEITO EM: 04/05/2008

Endereço para correspondência:

Flávia Leães de Almeida

Rua Dr Pantaleão, 587 ap. 209

Santa Maria - RS

CEP: 97010-180

E-mail: flaviafono@yahoo.com.br 